Bull. Austral. Math. Soc.

20D30, 20D25, 20D15

VOL. 69 (2004) [457-464]

\title{
CENTRE AND NORM
}

\section{J.C. Beidleman, H. Heineken, ANd M. Newell}

We establish a correlation between the structures of a group of power automorphisms of some group and their mutual commutator subgroup and consider the consequences for the norm of a group, and for its capability.

\section{INTRODUCTION}

The norm of a group is the intersection of all normalisers of the subgroups of a group. It is known that the norm contains the centre of the group and is contained in its second centre $Z_{2}(G)$ (see Schenkman [13] and Kappe [9] ). The purpose of this paper is a more specified description. Before we are more precise about this we begin with a more general situation and look at power automorphisms of a group. In what follows, we define the commutator $[a, b]$ of two elements to be $a^{-1} b^{-1} a b$ while $[a, \gamma]$ for a group element $a$ and an automorphism $\gamma$ stands for $a^{-1} a^{\gamma}$. Moreover, by a result of Cooper [4], $a^{-1} a^{\gamma} \in Z(G)$ whenever $\gamma$ is a power automorphism of $G$. If a position of the commutator bracket is filled by a set, the subgroup generated by the corresponding bracket elements is meant. Now we are able to formulate the more general result.

THEOREM 1. Let $G$ be a p-group, where $p$ is a prime. Let $\Gamma$ be a group of power automorphisms of $G$ and assume that $[G, \Gamma]$ has finite exponent.

(i) If $p$ is odd, then $\Gamma$ is cyclic or $[G, \Gamma]$ is cyclic.

(ii) If $p=2$, then $\Gamma$ is a subgroup of a direct product of a cyclic group and a group of order 2 or $[G, \Gamma]$ is cyclic.

REMARK 1. Divisible Abelian $p$-groups show that the condition on the finite exponent is indispensible here.

Now the following result is a consequence.

THEOREM 2. Let $G$ be a p-group and denote by $N(G), Z(G)$ its norm, centre respectively. At least one of the following two statements is true:

(A) $N(G) / Z(G)$ is cyclic,

(B) $[G, N(G)]$ is cyclic.

Received 19th November, 2003

Copyright Clearance Centre, Inc. Serial-fee code: 0004-9727/04 \$A2.00+0.00. 
REMARK 2. If $G$ is a regular $p$-group, then $N(G) / Z(G)$ is cyclic. If $x \in N(G)$, for some $p$-power $m$ we have $x^{m} \in Z(G)$ and if $x^{-1} y x=y^{k}$, then $y$ is of order dividing $k^{m}-1$. By Cooper [4, p. 349] power automorphisms are universal in regular $p$-groups, in other words, if $y, z \in G$ there is an integer $r$ such that $x^{-1} y x=y^{r}$ and $x^{-1} z x=z^{r}$, and the highest power of $p$ dividing $r-1$ is independant of the choice of $r$. We obtain that $G$ is of finite exponent and that there is $r \in \mathcal{N}$ such that $x^{-1} g x=g^{r}$ for all $g \in G$. So $N(G) / Z(G)$ is mapped onto the multiplicative group $Z_{i}^{*}$, where $t=\exp (G)$. Notice that $r=-1$ is impossible since the $p$-group is nonabelian if $N(G) \neq Z(G)$, and for $p=2$ we have that $r-1$ must be divisible by 4 .

Examples of groups satisfying (A) and those satisfying (B) of Theorem 2 are given at the end of this paper.

A group $G$ is called capable, if there is a group $H$ such that $H / Z(H) \cong G$; such groups are considered in detail in $[1,2,3,5,6,7]$. The normal subgroup of $Z^{*}(G)$ of $G$ called the epicentre of $G$, plays a significant role in determining whether or not $G$ is capable: it is the intersection of all normal subgroups $N$ of $G$ such that $G / N$ is capable. Thus $Z^{*}(G)$ is the smallest normal subgroup of $G$ such that $G / Z^{*}(G)$ is capable (see [3, Corollary 2.2]). Therefore $G$ is capable if and only if $Z^{*}(G)=1$. Another definition is

$$
Z^{*}(G)=\cap\{\phi(Z(E)) \mid(E, \phi) \text { is a central extension of } G\}
$$

(see $[1,2,3,5]$ ). In Theorems 3 and 4 we find some groups $G$ satisfying $N(G) \neq Z(G)$ which are not capable. We use the subgroup $Z^{*}(G)$ to prove these results.

THEOREM 3. Assume that $G$ is a finite group. If $[G, N(G)]^{m}$ is cyclic and nontrivial for some $m$, then $G$ is not capable.

We need a statement on the exponent of $[G, N(G)]$ for the next statement.

THEOREM 4. Assume that $G$ is a p-group. If $\mathrm{C}(N(G))$ possesses a supplement $S$ in $G$ such that $Z(S) \notin \mathrm{C}(N(G))$ and $[G, N(G)]^{2} \neq 1$, then $G$ is not capable.

Corollary. Assume that $G$ is a p-group, where $p \neq 2$, and $G$ is a split extension of an Abelian group $A$ by a group $B$. If $[G, N(G)]^{2} \neq 1$ and one of the following conditions is satisfied:

(i) $B$ is Abelian,

(ii) $\mathrm{C}(A)=A$,

then $G$ is not capable.

Example (VI) shows that the condition $[G, N(G)]^{2} \neq 1$ can not be strengthened.

PROOFS.

Proof of Theorem 1: By a result of Cooper ([4, Theorem 2.2.1], see also [14, Theorem 1.5.2]), $[G, \Gamma] \subseteq Z(G)$.- Let $x \in G$. Then $[x, \Gamma]$ is contained in $\langle x\rangle$ and thus 
cyclic. Choose $g \in G$ such that $[g, \Gamma]$ has maximal order. Then $[g, \Gamma]=\langle[g, \alpha]\rangle$ for some $\alpha \in \Gamma$. We claim that $[G, \Gamma]=\langle[g, \alpha]\rangle$ when $\Gamma$ is not cyclic, or (for $p=2)$ is not a subgroup of a direct product of a group of order 2 and a cyclic group.

Consider now the group $[G, \alpha]$ and suppose that it is not cyclic. Then (by $[\mathbf{1 2}, 4.27$ p. 102]) $[G, \alpha]=\langle[g, \alpha]\rangle \times V$ for some subgroup $V$. From this direct factor decomposition it follows that $\langle x\rangle \cap\langle y\rangle=1$ whenever $[v, \alpha] \in\langle x\rangle$ and $[g v, \alpha] \in\langle y\rangle$, with $1 \neq[v, \alpha] \in V$. In particular, $\langle v\rangle \cap\langle g v\rangle=1$.

Let $F(\alpha)=\left\{h \in G \mid h^{\alpha}=h\right\}$. Then $F(\alpha)$ is a $\Gamma$-invariant normal subgroup of $G$ and $G / F(\alpha) \cong[G, \alpha]$ and $G=\langle F(\alpha), g, M\rangle$ with $[M, \alpha]=V$.

Let $\Phi(g)=\left\{\delta \in \Gamma \mid g^{\delta}=g\right\}$. For $\beta \in \Phi(g)$ and $v \in M$ we have

$$
[g v, \beta]=[v, \beta] \in\langle g v\rangle \cap\langle v\rangle=1 .
$$

Therefore $\Phi(g) \subseteq \Phi(v)$. Let $h \in F(\alpha)$ and $\beta \in \Phi(g)$. Then $[v h, \alpha]=[v, \alpha] \in\langle v h\rangle$ and $[g v h, \alpha]=[g v, \alpha] \in\langle g v h\rangle$. Consequently $\langle v h\rangle \cap\langle g v h\rangle=1$. But $[v h, \beta]=[h, \beta]=[g v h, \beta]$ and so $[h, \beta]=1$. Therefore $\beta$ fixes $\langle F(\alpha), g, M\rangle=G$ and $\Phi(g)=1$. Since $\Gamma / \Phi(g)$ is isomorphic to a subgroup of Aut $(g)$ it is cyclic (for $p$ odd) or a subgroup of a direct product of a group of order 2 and a cyclic group. Hence it follows that $[G, \alpha]=\langle[g, \alpha]\rangle$ $=[g, \Gamma]$ when $\Gamma$ is not of the form just mentioned.

We still have to show $\langle[g, \alpha]\rangle=[G, \Gamma]$. As a first step, assume the existence of $w \in G$ and $\delta \in \Gamma$ such that $[w, \delta] \neq 1$ and $\langle[w, \delta]\rangle \cap[g, \Gamma]=1$. We have $[w, \alpha] \in\langle w\rangle \cap[G, \alpha]=1$ and $[w g, \alpha]=[g, \alpha]$ while $[w g, \delta]=[w, \delta][g, \delta]=[w, \delta][g, \alpha]^{m}$ for suitable $m$. These two elements generate a noncyclic group and not both of them are contained in $\langle w g\rangle$, a contradiction. We deduce $[G, \delta]$ is cyclic for every $\delta \in \Gamma$ and $[G, \delta] \cap[G, \alpha] \neq 1$. Assume now the existence of $w$ and $\delta$ as before but with the less stringent condition $[w, \delta] \notin[G, \alpha]$. Then $\langle[w, \alpha]\rangle$ is a proper subgroup of $\langle[w, \delta]\rangle$ and of $\langle[g, \alpha]\rangle$, likewise $\langle[g, \delta]\rangle$ is a proper subgroup of $\langle[g, \alpha]\rangle$ and of $\langle[w, \delta]\rangle$. We obtain $\langle[w g, \alpha]\rangle=\langle[g, \alpha]\rangle$ and $\langle[w g, \delta]\rangle=\langle[w, \delta]\rangle$ contrary to the automorphisms being power automorphisms. We have obtained $[w, \delta] \in[G, \alpha]=\langle[g, \alpha]\rangle$ for all $w \in G$ and $\delta \in \Gamma$, in other words, $[G, \Gamma]$ is cyclic. This concludes the proof.

Proof of TheOREM 2: As indicated in the introduction, $N(G) \subseteq Z_{2}(G)$. We shall apply Theorem 1 for subgroups $W$ with $Z(G) \subseteq W \subseteq N(G)$.

As a first step we notice two things: (I) there are no nontrivial divisible subgroups in $Z_{2}(G) / Z(G)$ if $G$ is a $p$-group; (II) there are no nontrivial divisible subgroups in $Z(G)$ if $G$ is a $p$-group and $N(G) \neq Z(G)$.

For statement (I) assume otherwise and consider an element $y \in Z_{2}(G) \backslash Z(G)$ such that there are elements $y_{i} \in Z_{2}(G)$ such that $y_{i}^{p^{p}} Z(G)=y Z(G)$ for all $i \in \mathcal{N}$. Let $h$ be any element of order $p^{i}$ in $G$. Then $[h, y]=\left[h, y_{i}^{p^{i}}\right]=\left[h, y_{i}\right]^{p^{i}}=\left[h^{p^{i}}, y_{i}\right]=1$. We have shown $y \in Z(G)$, contrary to assumption. So statement (I) is true.

For statement (II), assume the existence of a Prüfer subgroup $P$ in $Z(G)$ and choose 
an element $x \in N(G) \backslash Z(G)$. There is an element $y \in G$ which is not centralised by $x$. Now $\langle P, y\rangle$ is Abelian, and there is an element $z$ such that $\langle P, y\rangle=\langle P, z\rangle=P \times\langle z\rangle$. The element $z$ does not centralise $x$ and the same is true for $z w$ where $w \in P$ is of the same order as $z$. Now $x^{-1} z x=z^{k}, x^{-1} z w x=(z w)^{m}$ and $x^{-1} w x=w$. By the direct product description this is only possible for $k=m=1$ contrary to the construction of $z$. This shows statement (II).

As a consequence we state, that $N(G) / Z(G)$ and $[G, N(G)]$ do not possess divisible subgroups other than the trivial group.

Let $W_{i} / Z(G)$ be the maximal subgroup of exponent $p^{i}$ of $N(G) / Z(G)$. If $N(G) / Z(G)$ is non-cyclic, all $W_{i} / Z(G)$ are non-cyclic. We apply Theorem 1 for $G$ and $W_{i} / Z(G)$ considered as a group of (inner) power automorphisms. We have to show that the prime 2 does no longer play an extra role here. In other words, we have to show that the quotient group $N(G) /(N(G) \cap \mathrm{C}(T))$ is cyclic for every cyclic subgroup $T$ of $G$ regardless of the prime. Assume that $T=\langle y\rangle$ is of order $2^{n}$ with $n>2$. Then there is no $x \in N(G)$ such that $x^{-1} y x=y^{-1}$ since otherwise $[x,[x, y]]=y^{4} \neq 1$ and $x \in N(G) \subseteq Z_{2}(G)$. So for $n>2, N(G) /(N(G) \cap \mathbf{C}(T))$ is isomorphic to a subgroup of $Z_{2^{n}}^{*}$ and does not contain -1 , so it must be cyclic. - For $n<3$ we have that $Z_{2^{n}}^{*}$ is itself cyclic.

Using Theorem 1 for $G$ and $W_{i} / Z(G)$ with the above modification we obtain that $\left[G, W_{i}\right]$ is cyclic if $W_{i} / Z(G)$ is non-cyclic, its order is $p^{i}$. Since $[G, N(G)]$ can not be locally cyclic we obtain that $[G, N(G)]=\cup\left[G, W_{i}\right]$ is cyclic (and finite) and $N(G) / Z(G)$ $=\cup W_{i} / Z(G)$ must have finite exponent.

Proof of THEOREM 3: By hypothesis,

$$
T=[G, N(G)]^{m}=\left[G,(N(G))^{m}\right]
$$

is cyclic and nontrivial. We may assume that $T$ is of order a prime $p$ and that $T=\langle[h, d]\rangle$ with $h \in G$ and $d \in(N(G))^{m}$, so $d^{p} \in Z(G)$ and $T=[d, G]$. Now $C(d)$ is a normal subgroup of $G$ and $G / C(d)$ is cyclic of order $p$. There is a generating set $\left\{g_{1}, \ldots, g_{k}\right\}$ for $G$ such that $\left\langle g_{i}, C(d)\right\rangle=G$ for all $i$. But then $\left\langle\left[g_{i}, d\right]\right\rangle=T \subseteq\left\langle g_{i}\right\rangle$ for all $i$, and $T \subseteq Z^{*}(G)$ (see [3, Proposition 3.3]). So $G$ is not capable.

REMARK. A somewhat different proof of Theorem 3 can be given using [10, Theorem 2 and Corollary 1(2)].

Proof of Theorem 4: We shall show that $Z^{*}(G) \neq 1$ is true by $[N(G), Z(S)]$ $\subseteq Z^{*}(G)$. Let $G \cong H / M$ where $M \subseteq Z(H)$. For every $g \in G$ we choose some $g^{*} \in H$ as a pre-image of $g$ - since we argue by commutators the actual choice of this pre-image does not matter. Choose $d \in N(G)$ and $t \in Z(S)$ such that $[d, t] \neq 1$. We shall show $[d, t]^{2} \in Z^{*}(G)$. Since $d \in N(G)$, we have $[d, g] \in\langle g\rangle$ for all $g \in G$, and so $\left[\left[d^{*}, g^{*}\right], g^{*}\right]=1$, also $d^{*} \in Z_{3}(H)$. So $d^{*}$ is a right-2-Engel element of $H$. Let $s \in S$ and $c \in \mathbf{C}(N(G))$. By Robinson [11, Theorem 7.13](see also Kappe [9]) we have

$$
1=\left[\left[d^{*}, c^{*}\right], t^{*}\right]=\left[\left[d^{*}, t^{*}\right], c^{*}\right]^{-1}
$$


and

$$
1=\left[d^{*},\left[t^{*}, s^{*}\right]\right]=\left[\left[d^{*}, t^{*}\right], s^{*}\right]^{2} .
$$

We have shown $\left[d^{*}, t^{*}\right]^{2} \in Z(H)$ since $G=S C(N(G))$, and this proves Theorem 4 .

PROOF OF THE COROLLARY: It suffices to show that Theorem 4 may be applied: In both cases we take $S=A$ and we shall show $B \subseteq \mathbf{C}(N(G))$. Notice $G^{\prime} \subseteq A$ in the first case and $Z(G) \subseteq A$ in the second, so we have for both cases $G^{\prime} \cap Z(G) \subseteq A$. Now $[B, N(G)] \subseteq B \cap G^{\prime} \cap Z(G)=1$; so the hypotheses of Theorem 4 are satisfied, and the Corollary is true.

\section{EXAMPLES.}

(I) $N(G) / Z(G)$ is noncyclic.

The most prominent example is $Q_{8}$ for the prime 2. This is the member of smallest order of a family of examples which we shall now construct.

Let $\Gamma$ be a field of $p^{n}$ elements where $n=p^{k}$ and let $\gamma \in \Gamma$ be such that $\gamma^{p}-\gamma=\delta \neq 0$. We define the unitriangular matrices $M\left(\beta_{1}, \beta_{2}, \ldots, \beta_{p}\right)=\left(m_{i j}\right)$ such that

$$
\begin{array}{rlrl}
1 & \leqslant i, j \leqslant p+1, \\
m_{i j} & =0 & & \text { for } j<i, \\
m_{i i} & =1 & & \text { for all } i, \\
m_{i, k+i} & =\beta_{k}^{p^{i-1}} & & \text { for } k>0 .
\end{array}
$$

This set of $(p+1) \times(p+1)$-matrices forms a group with respect to usual matrix multiplication; we denote it by $T$. Let $\Phi$ be a maximal subgroup of $\Gamma^{+}$which contains neither 1 nor $\delta$. The set $\{M(0, \ldots, \mu) \mid \mu \in \Phi\}$ is a proper subgroup of $Z(T)$, we shall denote it by $F$.- The group $G=T / F$ is our example. We notice that $M\left(\beta_{1}, \ldots\right)^{n}=M\left(0, \ldots, 0, \beta_{1}^{a}\right)$ where $a=1+p+\cdots+p^{n-1}$. We see that $\beta_{1}^{a}$ is nonzero whenever $\beta_{1}$ is nonzero, and that it belongs to the prime field of $\Gamma$, so it does not belong to $\Phi$.

On the other hand we have $\left[M\left(\beta_{1}, \ldots\right), M(0, \ldots, 0, \lambda, \rho)\right]=M(0, \ldots, 0, \tau)$ where $\tau=\beta_{1} \lambda^{p}-\beta_{1}^{p^{n-1}} \lambda$, so for $\gamma^{p}=\beta_{1} \lambda^{p}$ we have $\tau=\delta \notin \Phi$. With the canonical epimorphism of $T$ onto $T / F=G$ we obtain that $N(G)=Z_{2}(T) / F=Z_{2}(G)$.

By Theorem 3, all of these groups are not capable. - Notice that the nilpotency class must be at least $p$ by [4]. In the second case we do not have such a restriction.

(II) $[G, N(G)]$ is noncyclic.

Let $p$ be a prime. Again we begin with a field $\Gamma$, this time of order $p^{n}$ where $2<n+1<p^{k}$. Form the unitriangular $(n+2) \times(n+2)$ - matrices over $\Gamma$ analogous to the examples in the previous section; notice that $\beta_{1}=\beta_{1}^{p^{n}}$ so $m_{12}=m_{n+1, n+2}$. Choose $\gamma \in \Gamma$ such that $\delta=\gamma^{p}-\gamma \neq 0$; for $n=2 ; p \neq 2$ take $\gamma$ such that $\gamma^{p}=-\gamma$ In particular, $\left[M\left(\beta_{1}, \ldots\right), M(0, \ldots, 0, \gamma, \mu)\right]=M\left(0, \ldots, 0, \delta \beta_{1}\right)$. We call the group of matrices $T$ as in the previous case. 
Let $A$ be the direct product of $n$ cyclic groups of order $p^{k+1}$. Then $A / A^{p}$ and $A^{p^{k}}$ are isomorphic to $\Gamma^{+}$, and we define these isomorphisms $\phi, \psi$ such that $\phi\left(a A^{p}\right)$ $=\psi\left(a^{p^{k}}\right)$. In the direct product $T \times A$ consider the subgroup generated by the elements $\left(M\left(\phi\left(a A^{p}\right), 0, \ldots, 0\right), a\right)$ and call it $U$. By construction, $U^{p}=\left\{\left(E, a^{p}\right)\right\}$ and $U_{n+1}$ $=\left\{\left(T_{n+1}, 1\right)\right\}$. Let

$$
V=\left\{\left(M\left(0, \ldots, 0, \delta \psi\left(a^{p^{k}}\right)\right), a^{p^{k}}\right)\right\} .
$$

Now $U / V=G$ is the desired group, and

$$
N(G)=\langle(M(0, \ldots, 0, \gamma, 0), 1) V, Z(G)\rangle .
$$

For $n=2$ and $\Gamma$ a prime field of odd order, $T$ contains a subgroup which is free (of exponent $p \neq 2$, of nilpotency class 3 and generated by two elements). This leads to a group $K$ of order $p^{5}$ with $N(K)=Z_{2}(K)$, see also (V).

Groups of class 2 we find for instance in the next family of examples (with $A$ of exponent $p^{2}$ and $B$ of order $p$ ).

(III) Iwasawa groups.

An Iwasawa group is a group in which all of its subgroups are permutable. For a complete description of finite Iwasawa $p$-groups see [14, p. 55]. Choose an Abelian group $A$ of exponent $p^{n}$ for $p \neq 2$ and $n>1$. Then form the split extension by a cyclic group $\langle b\rangle$ of order $p^{n-1}$ such that $b^{-1} a b=a^{1+p}$ for all $a \in A$. Then the element $c$ of order $p$ in $\langle b\rangle$ belongs to the norm of this extension. By the Corollary following Theorem 4, this group is not capable. For $p=2$ we choose $n>3$ and assume that the element $b$ of order $2^{n-2}$ maps every $a \in A$ onto $a^{5}$. In this case the element $c$ of order 4 in $\langle b\rangle$ is contained in the norm and again $[N(G), G]^{2} \neq 1$. Again this group is not capable. But if. $p=2 ; n=3 ; b=c$, then this group is in fact capable, see (VI).

(IV) Groups of maximal class.

The quotient group $\left(C_{p^{n}} \mathrm{wr} C_{p}\right) / Z\left(C_{p^{n}} \mathrm{wr} C_{p}\right)$ is a group of maximal class with unique Abelian normal subgroup of index $p$ which we call $N$. The subgroup of order $p$ operating on $N$ we denote by $\langle x\rangle$. Now extend $N$ by a element $y$ of order $p^{2}$ satisfying the following conditions: $y^{-1} a y=x^{-1} a x$ for all $a \in N$, and $y^{p} \in Z(\langle N, y\rangle)$. Then $Z_{2}(\langle N, y\rangle)$ $=N(\langle N, y\rangle)$ and Wielandt series and central series coincide in all other places. Again, this group is not capable by Theorem 3 . The same applies for all of its nonabelian subgroups. Notice that we have the family of (generalised) quaternion groups here if $p=2$.

(V) Two-generator groups of class 3 .

Choose a prime $p>3$ and define

$$
D=\left\langle x, y \mid x^{p}=[[x, y], x] ; y^{p}=[[x, y], y] ;[[[x, y], x], y]=1\right\rangle .
$$

The relations yield

$$
[[[x, y], x], x]=1=[[[x, y], y], y]
$$


and

$$
[[[x, y], y], x]=1
$$

so $D$ is nilpotent of class 3 and $N(D)=D^{\prime}$, also $[D, N(D)]$ is of rank 2 . Here $D$ is not capable: If $D \cong H / Z(H)$ and $x^{*}, y^{*} \in H$ are the pre-images of $x, y$, then again

$$
\left[\left[\left[x^{*}, y^{*}\right], x^{*}\right], x^{*}\right]=1=\left[\left[\left[x^{*}, y^{*}\right], y^{*}\right], y^{*}\right]
$$

and

$$
\left[\left[\left[x^{*}, y^{*}\right], x^{*} y^{*}\right], x^{*} y^{*}\right]=1
$$

and so

$$
\left[\left[\left[x^{*}, y^{*}\right], x^{*}\right], y^{*}\right]\left[\left[\left[x^{*}, y^{*}\right], y^{*}\right], x^{*}\right]=1
$$

while

$$
1=\left[\left[x^{*}, y^{*}\right],\left[x^{*}, y^{*}\right]\right]=\left[\left[\left[x^{*}, y^{*}\right], x^{*}\right], y^{*}\right]\left[\left[\left[x^{*}, y^{*}\right], y^{*}\right], x^{*}\right]^{-1} \text {. }
$$

This shows that all commutators of lengh 4 are trivial and $D \cong H$ contrary to assumption.- For the prime 3 , the powers $x^{p}, y^{p}$ in the relations should be replaced by $x^{9}, y^{9}$ to obtain the same result.- An argument like that in the proof of Theorem 4 shows that these groups are not capable.

(VI) Capable 2- groups.

Let $s=2^{n}>2$ and $1 \leqslant i, j, k \leqslant m$ with $m \neq 1$. Consider

$$
T=\left\langle w_{1}, w_{2}, \ldots, w_{m}, v \mid 1=\left[\left[w_{i}, w_{j}\right], w_{k}\right]=w_{i}^{2 s}=v^{2}=w_{i}^{-s-1} v^{-1} w_{i} v\right\rangle
$$

The centre $Z(T)$ is generated by all commutators $\left[w_{i}, w_{j}\right]$, and

$$
N(T / Z(T))=\left\langle w_{i}^{2}, v, Z(T)\right\rangle / Z(T)
$$

This shows that $G$ may be capable if $[G, N(G)]^{2}=1$.

\section{REFERENCES}

[1] M.R. Bacon and L.C. Kappe, 'On capable p-groups of nilpotency class two.', Illinois J. Math. 47 (2003), 49-62.

[2] F.R. Beyl, U. Felgner and P. Schmid, 'On groups occurring as central factor groups', $J$. Algebra 61 (1979), 161-177.

[3] F.R. Beyl and J. Tappe, Group extensions, representations, and the Schur multiplicator, Lecture Notes in Mathematics 958 (Springer-Verlag, Berlin, Heidelberg, New York, 1982).

[4] C.D.H. Cooper, 'Power automorphisms of a group', Math. Z. 107 (1968), 335-356.

[5] G. Ellis, 'On the capability of groups.', Proc. Edinburgh Math. Soc. 41 (1998), 487-495.

[6] H. Heineken, 'Nilpotent groups of class 2 that can appear as central quotient groups.', Rend. Sern. Mat. Univ. Padova 84 (1990), 241-248. 
[7] H. Heineken and D. Nikolova, 'Class two nilpotent capable groups', Bull. Austral. Math. Soc. 54 (1996), 347-352.

[8] W. Kappe, 'Review of [13]', Math. Reviews 22 (1960), No. 8033.

[9] W. Kappe, 'Die A-Norm einer Gruppe', nlinois J. Math. 5 (1961), 187-197.

[10] M.L. Newell, 'Normal and power endomorphisms of a group.', Math. Z. 151 (1976), 139-142.

[11] D.J.S. Robinson, Finiteness conditions and generalized soluble groups, Ergebnisse der Mathematik und ihrer Grenzgebiete 63, Part 2 (Springer-Verlag, Berlin, Heidelberg, New York, 1972).

[12] D.J.S. Robinson, A course in the theory of groups, Graduate Texts in Mathematics, 2nd. edition (Springer-Verlag, New York, Heidelberg, Berlin, 1996).

[13] E. Schenkman, 'On the norm of a group', Ilinois J. Math. 4 (1960), 396-398.

[14] R. Schmidt, Subgroup lattices of groups, de Gruyter Expositions in Mathematics 14 (Walter de Gruyter, Berlin, 1994).

Department of Mathematics

University of Kentucky

Lexington, KY 40506-0027

United States of America

Department of Mathematics National University of Ireland

Galway

lreland
Mathematisches Institut

Universität Würzburg

Am Hubland

97074 Würzburg

Germany 